\title{
Medikamentöse Systemtherapien der Psoriasis
}

\section{Systemic Drug Therapy in Psoriasis}

R. Mössner, M. P. Schön

Abteilung für Dermatologie und Venerologie, Georg-August-Universität Göttingen

\section{Lernziele}

$\nabla$

Kenntnisse über

- Eigenschaften, Wirksamkeit und Sicherheitsaspekte von klassischen Systemtherapeutika und Biologika

- systemische Therapie in Schwangerschaft und Stillzeit

- Systemtherapien bei Männern mit Kinderwunsch

- Therapiekosten

\section{Einleitung \\ $\nabla$}

Die zur Behandlung der Psoriasis zugelassenen Systemtherapeutika lassen sich in 2 Gruppen einteilen. Zur ersten Gruppe zählen die zur FirstLine-Therapie zugelassenen sogenannten klassischen Systemtherapeutika Cyclosporin A (CyA), Fumarsäureester, Methotrexat (MTX) und Retinoide (Acitretin), zur zweiten Gruppe gehören die bisher nur als Second-Line-Therapeutika zugelassenen Biologika. Die Gruppe der Biologika umfasst aktuell die TNF-Antagonisten (TNF = Tumor-Nekrose-Faktor) Adalimumab, Etanercept und Infliximab sowie den gegen CD11a gerichteten Antikörper Efalizumab.

VNR

2760512010047430464

Bibliografie

Dol http://dx.doi.org/

10.1055/s-0029-1214474

Online-Publikation: 27.4. 2009

Akt Dermatol 2010; 36:

142-152 @ Georg Thieme

Verlag KG Stuttgart · New York ISSN 0340-2541

Korrespondenzadresse PD Dr. med. Rotraut Mössner Abteilung für Dermatologie und Venerologie

Georg-August-Universität

Von-Siebold-Str. 3

37075 Göttingen

rmoessn@gwdg.de
Im Folgenden werden wichtige Eigenschaften, Indikationsgebiete sowie Anwendungsbeschränkungen der Systemtherapeutika dargestellt. Aufgrund der Fülle der verfügbaren Informationen wurde bei der Darstellung der einzelnen Medikamente ein Schwerpunkt auf praktisch relevante Aspekte gelegt. Für eine umfassende Darstellung wird auf die jeweiligen Fachinformationen sowie

\footnotetext{
* Nach Drucklegung dieses Artikels hat die EMEA das Aussetzen der Zulassung von Raptiva ${ }^{\circledR}$ (Efalizumab) empfohlen. Grund hierfür ist eine geänderte Bewertung des Nutzen-/Risiko-Verhältnisses, nachdem drei bestätigte Fälle und ein Verdachtsfall einer progressiven multifokalen Leukenzepholapathie (PML) augetreten sind. Die drei Psoriasis-Patienten mit bestätigter PML hatten Efalizumab für einen Zeitraum von über 3 Jahren erhalten.
}

Biologika sind bei der Psoriasis nur als Second-LineTherapie zugelassen.

auf die aktuelle S3-Leitlinie zur Therapie der Psoriasis vulgaris verwiesen [1].

Bei Medikamenten, die als Monopräparate verfügbar sind, geben wir den Wirkstoff an, während wir bei Kombinationspräparaten den Handelsnamen verwenden.

PASI. Der Psoriasis-Area-and-Severity-Index (PASI) spielt bei den meisten Studien zur Evaluation der Effektivität und auch in der Praxis bei der Indikationsstellung zur Systemtherapie bei Patienten eine große Rolle und soll daher kurz dargestellt werden. Der PASI berücksichtigt die Ausprägung der Symptome Rötung, Infiltration und Schuppung sowie die Ausdehnung der Erkrankung in den verschiedenen Körperregionen. Es können Werte zwischen 0 (erscheinungsfrei) und 72 (maximaler Schweregrad) erreicht werden. Ab einem PASI von 10 spricht man von einer mittelschweren, ab einem Wert von 15 von einer schweren Psoriasis vulgaris. Zur Bewertung der Effektivität einer Psoriasistherapie wird in klinischen Studien inzwischen meist der prozentuale Anteil der Patienten angegeben, der eine mindestens 75\%ige Besserung des PASI relativ zum Ausgangsbefund (PASI75) erzielte. Auch bei der Beurteilung des Therapieerfolgs in der Praxis kann der PASI herangezogen werden. Hier ist 3-4 Monate nach Beginn einer Systemtherapie eine PASI75-Antwort wünschenswert, als mindestens zu erreichender Schwellenwert wird in der Regel eine $50 \%$ ige Besserung des PASI (PASI50) in diesem Zeitraum angesehen.

Definition der PASI-75 Antwort: Eine mindestens $75 \%$ ige Besserung des PASI relativ zum Ausgangsbefund. 


\section{Klassische Systemtherapeutika} $\nabla$

\section{Fumarsäureester}

Fumarsäureester sind kleine Moleküle aus der Gruppe der Dikarbonsäuren. Fumarsäureester sind als Kombinationspräparate verfügbar, die Ethylhydrogenfumarat und Dimethylfumarat, das als pharmakologisch aktiver Bestandteil gilt, enthalten. Hierbei enthält Fumaderm ${ }^{\circledR}$ initial mit 30 mg Dimethylfumarat ein Viertel der Menge an Dimethylfumarat, die in Fumaderm ${ }^{\circledR}$ enthalten ist $(120 \mathrm{mg})$, während die enthaltene Menge an Ethylhydrogenfumarat in beiden Präparaten identisch ist. Fumaderm ${ }^{\circledR}$ ist wahrscheinlich das in Deutschland gegen die Psoriasis vulgaris am häufigsten eingesetzte Systemtherapeutikum [2].

Fumarsäureester wirken immunmodulierend, antientzündlich, antiproliferativ und apoptotisch auf aktivierte T-Zellen. Es wird davon ausgegangen, dass der hauptsächliche Wirkmechanismus von Dimethylfumarat in einer Beeinflussung des intrazellulären Glutathions besteht. Das Glutathion-System ist eines der wichtigsten intrazellulären Systeme zur Entgiftung toxischer Sauerstoffmetabolite und freier Radikale und die Verschiebung des Redox-Gleichgewichts beeinflusst u. a. entzündungsfördernde Kaskaden.

\section{Wirksamkeit}

Fumaderm ${ }^{\circledR}$ wird oral verabreicht. Da bei den öfter auftretenden gastrointestinalen Nebenwirkungen (s. folgender Abschnitt) ein Gewöhnungseffekt eintreten kann, wird Fumaderm ${ }^{\circledR}$ langsam aufdosiert. Hierbei wird zuerst Fuma$\operatorname{derm}^{\circledR}$ initial wöchentlich um eine Tablette täglich auf 3 Tabletten/Tag gesteigert, anschließend erfolgt die Therapie mit Fumaderm ${ }^{\circledR}$, dessen Dosierung ebenfalls wöchentlich um eine Tablette auf bis zu maximal 6 Tabletten/Tag gesteigert wird. Eine Metaanalyse klinischer Studien ergab, dass unter Therapie mit Fumaderm ${ }^{\circledR}$ nach 16 Wochen ein PASI75 bei 50\%-70\% der Patienten erreicht wird [1]. In einer offenen Langzeitstudie konnte gezeigt werden, dass es unter fortgesetzter Therapie mit Fumaderm ${ }^{\circledR}$ über den Zeitraum von einem Jahr zu einer weiteren kontinuierlichen Verbesserung der Psoriasis kam. Die mittlere Verbesserung des PASI nach einem Jahr im Vergleich zum Ausgangsbefund lag bei 76\% [3].

\section{Sicherheitsaspekte}

Unter einer Fumaderm ${ }^{\circledR}$-Therapie treten gastrointestinale Nebenwirkungen wie Diarrhoe, Magenschmerzen und Oberbauchkrämpfe häufig während der ersten Therapiewochen auf. Diese Beschwerden bessern sich häufig im Therapieverlauf, worauf die Patienten hingewiesen werden sollten. Ferner kann es zu einer Flush-Symptomatik mit anfallsartiger Rötung des Gesichts, seltener auch anderer Körperareale kommen. Ferner können Blutbildveränderungen auftreten, wie eine meist vorübergehende Eosinophilie (vor allem während der ersten 3 Therapiemonate), eine Granulozytopenie sowie eine eher bei Langzeittherapie auftretende Lymphopenie, wobei hier eine absolute Lymphozytenzahl $<500 / \mu l$ als Abbruchkriterium für die Fumaderm ${ }^{\circledR}$-Therapie angesehen wird. Seltener kommt es zum Anstieg der Leberwerte und zu Funktionsstörungen der Niere. Arzneimittelinteraktionen mit anderen Medikamenten sind nicht bekannt. Insgesamt besteht bei der Fumaderm ${ }^{\circledR}$-Therapie ein günstiges Nutzen-Risiko Verhältnis.

Eine absolute Lymphopenie tritt vor allem in der Langzeittherapie mit Fumaderm ${ }^{\circledR}$ auf.

\section{Acitretin}

Acitretin ist ein Vitamin-A-Säure-Derivat, das an Retinsäure-Rezeptoren (RAR) $\alpha, \beta$ und $\gamma$, nukleäre Transkriptionsfaktoren, bindet, diese dadurch aktiviert und so die Expression verschiedener Gene beeinflusst. Es besitzt antiproliferative Eigenschaften und kann bei Psoriasis-Patienten die Hyperproliferation und den Differenzierungsverlust der epidermalen Keratinozyten normalisieren. Ferner wirkt Acitretin immunmodulierend.

\section{Wirksamkeit}

Acitretin wird oral verabreicht. In der S3-Leitlinie werden tägliche Anfangsdosen zwischen 0,3 und 0,5 mg/kg KG empfohlen, mit konsekutiver Anpassung der Dosis, wobei hier üblicherweise Dosen zwischen 0,5 und $0,8 \mathrm{mg} / \mathrm{kg}$ täglich Einsatz finden. Die Wirkung ist stark dosisabhängig. In einer 8-wöchigen, doppelblinden, placebokontrollierten Studie zeigte sich bei Dosierungen zwischen 10 und $25 \mathrm{mg} /$ Tag keine Wirkung, während im Dosisbereich zwischen 50 und $75 \mathrm{mg} /$ Tag 25\% der Patienten einen PASI75 erreichten [4]. Zur Wirkungssteigerung wird Acitretin häufig mit einer PUVA- oder UVB-Therapie kombiniert.

\section{Sicherheitsaspekte}

Acitretin ist hochgradig teratogen. Da Acitretin (Halbwertszeit etwa 2 Tage) insbesondere unter Alkoholeinfluss in Etretinat mit einer Halbwertszeit von 120 Tagen umgewandelt werden kann, darf unter Acitretin und auch 2 Jahre nach Beendigung der Acitretin-Therapie keine Schwangerschaft eintreten. Aufgrund dieses langen Zeitraums sollte Acitretin höchstens in Ausnahmefällen bei Frauen im gebärfähigen Alter eingesetzt werden; hier ist dann Alkoholabstinenz während der Therapie und in den ersten 2 Monaten nach Therapieende besonders wichtig. Obligate Nebenwirkungen von Acitretin sind eine Xerosis der Haut und der Schleimhäute einschließlich einer Cheilitis (eine Cheilitis ist ein Indikator für eine Dosierung im therapeutischen Bereich). Es kann ferner zu reversibler diffuser Alopezie sowie zu

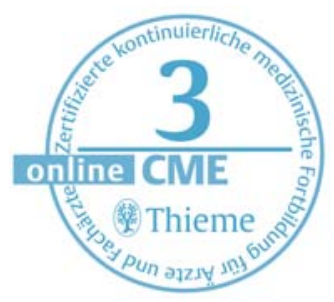


Abb. 1 Hypertrichose be einer 55-jährigen PsoriasisPatientin unter CyA (4 mg/ $\mathrm{kg} \mathrm{KG})$.

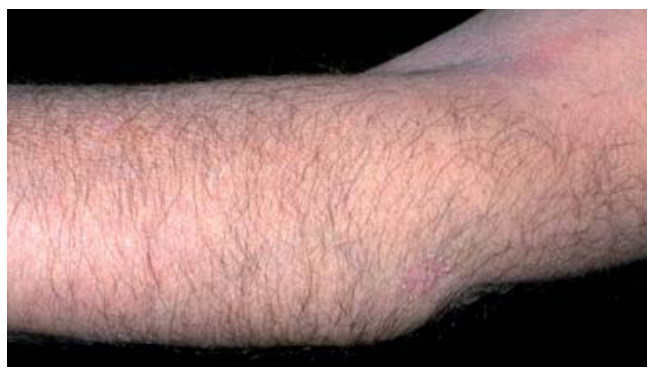

Bindehautentzündung kommen (daher Therapie bei Kontaktlinsenträgern eingeschränkt). Manchmal wird ,Schleiersehen' angegeben, ohne dass bei ophthalmologischer Untersuchung ein organisches Korrelat gefunden wird. Ferner bewirkt Acitretin häufig eine Erhöhung der Serumlipide und/oder Leberwerte. Muskel- und Knochenschmerzen können auftreten, und bei Langzeittherapie mit Acitretin können Skelettabnormalitäten ähnlich einer diffusen idiopathischen skelettalen Hyperostose mit Osteophytenbildung auftreten. Hirndrucksteigerungen unter einer Monotherapie mit Acitretin wurden beobachtet, die Gefahr hierfür ist aber deutlich höher, wenn Retinoide mit Tetrazyklin kombiniert werden, sodass derartige Kombinationen kontraindiziert sind. Bei Acictretin ist ferner relevant, dass die Wirkung niedrig dosierter Progesteron-Pillen vermindert werden kann.

Acitretin sollte höchstens in Ausnahmefällen bei Frauen im gebärfähigen Alter eingesetzt werden. Alkoholabstinenz ist unerlässlich.

Aufgrund der potenziell schwerwiegenden teratogenen Wirkungen von Acitretin empfiehlt sich eine schriftliche Aufklärung. Auch muss darauf hingewiesen werden, dass während und ein Jahr nach der Therapie kein Blut gespendet werden darf.

Auch wenn unter Acitretin häufig störende Nebenwirkungen auftreten, sind diese nach Therapieende meist vollständig reversibel. Es wird trotz seiner in klinischen Studien eher mäßigen Wirkung weiterhin eingesetzt, und stellt vor allem in Kombination mit UV-Therapie sowie bei Patienten, bei denen immunsuppressive Therapien kontraindiziert sind, eine Therapiealternative dar.

\section{Cyclosporin A}

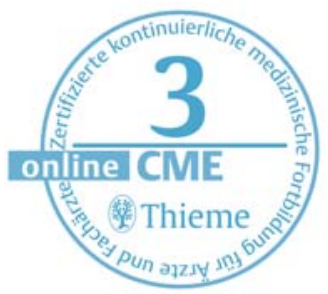

rem die Aktivität des nukleären Transkriptionsfaktors NFAT verringert, der wesentlich an der Transkription pro-inflammatorischer Zytokine wie IL-8 und TNF- $\alpha$ in T-Zellen beteiligt ist.

\section{Wirksamkeit}

CyA wird in einer Dosierung zwischen 3 und $5 \mathrm{mg} / \mathrm{kg} \mathrm{KG}$ oral verabreicht. Empfohlen wird entweder eine Intervalltherapie über 8 bis 16 Wochen, wobei CyA in der Regel ausschleichend abgesetzt wird, oder eine Langzeittherapie, deren Dauer 2 Jahre nicht übersteigen sollte. Die Wirkung von CyA ist dosisabhängig. Eine kürzlich veröffentliche 12-wöchige, randomisierte, offene klinische Studie mit 68 Patienten, die CyA (3 mg/ $\mathrm{kg} \mathrm{KG}$, Dosiserhöhung auf $5 \mathrm{mg} / \mathrm{kg} \mathrm{KG}$ möglich) mit MTX (7,5 mg/Woche, Dosiserhöhung auf $15 \mathrm{mg} /$ Woche möglich, plus $5 \mathrm{mg}$ Folsäure täglich außer am Tag der MTX-Gabe) in der Therapie der Plaque-Psoriasis vergleicht, ergab in der CyAGruppe eine PASI75-Antwort von 58\% nach 12 Wochen vs. 24\% in der MTX-Gruppe [5].

\section{Sicherheitsaspekte}

Typische Nebenwirkungen von CyA sind Nierenfunktionsstörungen, die auch irreversibel sein können, und das Auftreten oder die Verschlechterung einer arteriellen Hypertonie. Häufig treten Müdigkeit, Kopfschmerzen, Parästhesien, Hyper-

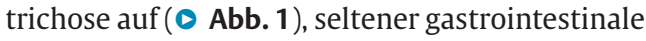
Beschwerden sowie Gingivahyperplasie, die durch Nifedipin verschlechtert werden kann. Ferner können Hyperlipidämie, Hyperkaliämie und Hypomagnesiämie auftreten. Unter CyA kann es ferner vermehrt zu Infektionen kommen. CyA scheint auch eine erhöhte Malignomrate zu bedingen, insbesondere von malignen Hauttumoren bei Patienten mit vorangegangener PUVATherapie. Die Ergebnisse der bereits oben erwähnten Studie ergeben einen guten Aufschluss über die Nebenwirkungen von CyA im Vergleich mit MTX während der Induktionsphase. Während Hypertrichose (13\%), Muskelkrämpfe (13\%), Myalgien (16\%), Parästhesien (35\%) und Kreatininanstieg (19\%) lediglich in der mit CyA therapierten Patientengruppe auftraten, und in der CyA-Gruppe Fatigue mit 48\% (MTX: 16\%) und Kopfschmerzen mit 29\% (MTX: 14\%) deutlich häufiger auftraten als in der MTX-Gruppe, traten Leberwerterhöhungen nur in der MTX-Gruppe auf (19\%).

Wechselwirkungen. Ein wesentlicher Sicherheitsaspekt von CyA sind Wechselwirkungen mit anderen Medikamenten, die auf mehreren Ebenen stattfinden können. CyA wird über das hepatische Enzym Cytochrom-P450-3A4 (CYP3A4) metabolisiert. So können Hemmer der CYP3A4-Aktivität wie Allopurinol, Kalziumantagonisten, Makrolidantibiotika und Ketokonazol, aber auch Grapefruitsaft zu einer Erhöhung des CyA-Spiegels führen, während Induktoren von CYP3A4 wie Carbamazepin oder johanniskraut- 
haltige Präparate den CyA-Spiegel senken können. CyA kann ferner die Clearance anderer Medikamente wie Digoxin verringern und es vermindert die Wirkung progesteronhaltiger Kontrazeptiva. Ferner muss auf eine mögliche Verstärkung der Nephrotoxizität anderer Medikamente geachtet werden.

Die Wirkung progesteronhaltiger Kontrazeptiva kann unter CyA vermindert sein.

\section{Methotrexat}

Methotrexat (MTX) ist das weltweit am häufigsten zur Therapie der Psoriasis eingesetzte Systemtherapeutikum. Es ist ein strukturelles Analog der Folsäure und wirkt als Folsäureantagonist. Über diesen Mechanismus hemmt MTX die De-novoSynthese von Purin und Pyrimidin und wirkt so antiproliferativ. Ferner besitzt MTX anti-inflammatorische und immunmodulierende Wirkungen, deren zugrunde liegende Mechanismen noch nicht vollständig geklärt sind.

\section{Wirksamkeit}

MTX wird einmal wöchentlich oral oder parenteral (meist s.c., seltener i.m. oder i.v.) verabreicht. $\mathrm{Zu}$ Beginn der Therapie erfolgt entweder eine langsame Dosissteigerung, beginnend bei $5-7,5 \mathrm{mg} /$ Woche, es kann aber auch sofort mit einer höheren Dosis begonnen werden. Die Dosierung von MTX sollte in der Regel 22,5 mg/Woche nicht überschreiten. Mit dem Ziel, die Nebenwirkungen zu reduzieren, wurde empfohlen, bei oraler Gabe die Dosis auf 3 Einzeldosen im Abstand von jeweils $12 \mathrm{~h}$ zu verteilen. Da der Nutzen dieser Maßnahme bisher nicht nachgewiesen wurde, wird MTX inzwischen auch oral häufig als Einzeldosis verabreicht. Zur Reduktion gastrointestinaler Nebenwirkungen wird die Gabe von Folsäure empfohlen. Die hier verwendeten Dosierungen bewegen sich zwischen $5 \mathrm{mg}$ einmal wöchentlich am Tag nach der MTX-Gabe und 1-5 mg an allen MTX-freien Tagen.

PASI75-Antworten unter MTX-Therapie werden in der aktuellen S3-Leitlinie mit etwa $60 \%$ am Ende der Induktionsphase angegeben [1]. In einer kürzlich veröffentlichten offenen klinischen Studie, in der MTX bis zu einer Dosis von $15 \mathrm{mg} /$ Woche als orale Gabe aufdosiert werden konnte, zeigten allerdings nur 24\% der Patienten nach 12 Wochen eine PASI75-Antwort [5], in einer kontrollierten Studie, in der MTX bis zu einer Dosis von $25 \mathrm{mg} /$ Woche aufdosiert werden konnte, betrug die PASI75-Antwort nach 16 Wochen $36 \%$ [6].

\section{Sicherheitsaspekte}

Häufige Nebenwirkungen einer MTX-Therapie sind Übelkeit, reversibler Haarausfall und leichte Leberwerterhöhungen. Ferner kann es zu Kopf- schmerzen und zu Ulzerationen der Mundschleimhaut und des Gastrointestinaltrakts kommen, wobei bei Auftreten mukosaler Ulzerationen häufig eine versehentliche Überdosierung von MTX stattgefunden hat. Ferner können unter MTX vermehrt Infektionen auftreten. Besonderes Augenmerk sollte auf die potenziell letalen Nebenwirkungen einer (schweren) Knochenmarksuppression mit Leukopenie, Thrombozytopenie oder Panzytopenie gerichtet werden. Eine weitere schwere, wenngleich sehr seltene Nebenwirkung ist die interstitielle Pneumonitis oder Alveolitis, die sich mit trockenem Husten, Fieber und Dyspnoe äußern kann. Treten letztgenannte Symptome bei einem Patienten unter MTX auf, soll frühzeitig eine Abklärung erfolgen und die MTX-Therapie unterbrochen werden.

Hepatotoxizität. Eine wichtige Nebenwirkung der MTX-Therapie ist die Hepatotoxizität, die vor allem bei Langzeittherapie von Bedeutung ist, wobei es hier zu Leberfibrose und Leberzirrhose kommen kann. Angaben zur Häufigkeit des Auftretens einer Leberzirrhose variieren zwischen 0 und $25 \%$, wobei Alkoholkonsum, Adipositas, Hepatitis, Diabetes mellitus und positive Familienanamnese für Lebererkrankungen als Risikofaktoren gelten. Als Screeningverfahren für eine Leberfibrose wird die Verlaufsbestimmung des aminoterminalen Propeptides von Typ-III-Prokollagen empfohlen, wobei hier prospektive Langzeituntersuchungen an großen Kollektiven noch ausstehen. Während früher eine regelmäßige Leberblindpunktion im Therapieverlauf nach jeder Kumulativdosis von 1,5 g (also etwa alle 2 Jahre) empfohlen wurde, ist diese Empfehlung inzwischen gelockert worden. Praktisch bedeutet dies, dass derzeitig die Indikation zur Leberbiopsie im Einzelfall unter Zusammenschau von serologischen Parametern, Therapiedauer, Risikofaktoren und bildgebenden Verfahren möglichst durch einen Internisten oder Hepatologen gestellt werden sollte.

Trockener Husten, Fieber und Dyspnoe unter MTXTherapie können ein Hinweis auf eine gefährliche interstitielle Pneumonitis oder Alveolitis sein. Gastrointestinale Ulzerationen sind häufig durch MTXÜberdosierung bedingt.

\section{Biologika}

Der Begriff Biologika (Biologic) geht auf die Kurzform von Biological Products zurück. Er bezeichnet Viren, therapeutische Seren, Toxine, Antitoxine oder analoge Produkte, die zur Prävention und Therapie von Erkrankungen oder Verletzungen bei Menschen eingesetzt werden können. Biologika, die chronisch entzündliche Prozesse modulieren können, zählen zu den Disease modifying antirheumatic Drugs (DMARD). Zur Therapie der

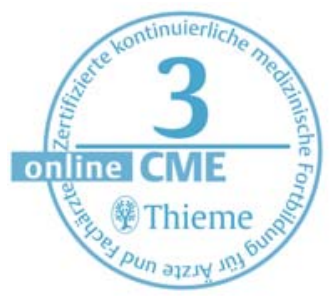


Tab. 1 Charakteristika der Biologika.

\begin{tabular}{|c|c|c|c|c|}
\hline & Infliximab & Etanercept & Adalimumab & Efalizumab \\
\hline Molekül & $\begin{array}{l}\text { chimärer } \operatorname{lgG}_{1} / \text { kappa } \\
\text { monoklonaler Antikörper }\end{array}$ & $\begin{array}{l}\text { p75-lgG } \mathrm{Fc}_{1} \mathrm{~F}-\text { Fragment } \\
\text { Fusionsprotein }\end{array}$ & $\begin{array}{l}\text { humaner } \mathrm{IgG}_{1} / \text { kappa } \\
\text { monoklonaler Antikörper }\end{array}$ & $\begin{array}{l}\text { humaner } \mathrm{IgG}_{1} / \text { kappa } \\
\text { monoklonaler Antikörper }\end{array}$ \\
\hline Zielmolekül & TNF- $\alpha$ & $\begin{array}{l}\text { TNF- } \alpha \\
\text { Lymphotoxin- } \alpha \text { (TNF- } \beta)\end{array}$ & TNF- $\alpha$ & $\begin{array}{l}\text { CD11a (Untereinheit von } \\
\text { LFA-1) }\end{array}$ \\
\hline Zulassung & $\begin{array}{l}\text { mittelschwere bis schwere } \\
\text { Plaque-Psoriasis } \\
\text { Psoriasisarthritis } \\
\text { (in Kombination mit MTX, } \\
\text { sofern möglich) }\end{array}$ & $\begin{array}{l}\text { mittelschwere bis schwere } \\
\text { Plaque-Psoriasis (Zulassung } \\
\text { nur für } 24 \text { Wochen) } \\
\text { Psoriasisarthritis }\end{array}$ & $\begin{array}{l}\text { mittelschwere bis schwere } \\
\text { Plaque-Psoriasis } \\
\text { Psoriasisarthritis }\end{array}$ & $\begin{array}{l}\text { mittelschwere bis schwere } \\
\text { Plaque-Psoriasis }\end{array}$ \\
\hline Dosierung & $\begin{array}{l}5 \mathrm{mg} / \mathrm{kg} \mathrm{KG} \text { intravenös } \\
\text { (i. v.) Woche } 0,2 \text { and } 6 \text {, } \\
\text { danach alle } 8 \text { Wochen }\end{array}$ & $\begin{array}{l}2 \times 25 \mathrm{mg} / \text { Woche subkutan } \\
\text { (s.c.) oder } 1 \times 50 \mathrm{mg} / \text { Woche } \\
\text { s.c. } \\
2 \times 50 \mathrm{mg} / \text { Woche möglich } \\
\text { während der ersten } 12 \text { Wo- } \\
\text { chen bei Plaque-Psoriasis }\end{array}$ & $\begin{array}{l}80 \mathrm{mg} \text { s.c. Woche } 0,40 \mathrm{mg} \\
\text { s. c. Woche } 1 \text {, danach } 40 \mathrm{mg} \\
\text { s. c. alle } 14 \text { Tage für Plaque- } \\
\text { Psoriasis } \\
40 \mathrm{mg} \text { s. c. alle } 14 \text { Tage für } \\
\text { Psoriasisarthritis }\end{array}$ & $\begin{array}{l}0,7 \mathrm{mg} / \mathrm{kg} \mathrm{KG} \mathrm{s.} \mathrm{c.} \mathrm{Woche} 0 \text {, } \\
\text { danach wöchentlich } 1 \mathrm{mg} / \mathrm{kg} \\
\text { KG s. c. }\end{array}$ \\
\hline Halbwertszeit & $\begin{array}{l}\text { Einfachgabe: } 8-9,5 \text { Tage } \\
\text { Steady State: mehrere } \\
\text { Wochen }\end{array}$ & $\begin{array}{l}\text { Einfachgabe: } \sim 70 \mathrm{~h} \\
\text { Steady State: } \sim 100 \mathrm{~h}\end{array}$ & Einfachgabe: 14 Tage & $\begin{array}{l}\text { Einfachgabe: } 6 \text { Tage } \\
\text { Steady State: } \sim 25 \text { Tage }\end{array}$ \\
\hline $\begin{array}{l}\text { Wirksamkeit bei } \\
\text { Psoriasis (PASI 75) }\end{array}$ & $\begin{array}{l}\text { Woche 10: } 80 \% \\
\text { Woche } 24: \sim 80 \%\end{array}$ & $\begin{array}{l}2 \times 25 \mathrm{mg} / \text { Woche: } \\
\text { Woche 12: } 34 \% \\
\text { Woche } 24: \sim 50 \% \\
1 \times 50 \mathrm{mg} / \text { Woche: } \\
\text { Woche } 12: 37 \% \\
\text { Woche } 24: 71 \% \\
2 \times 50 \mathrm{mg} / \text { Woche: } \\
\text { Woche } 12: \sim 50 \%\end{array}$ & $\begin{array}{l}\text { Woche } 16: 71-80 \% \\
\text { Woche } 24:-70 \%\end{array}$ & $\begin{array}{l}\text { Woche } 12: 22-39 \% \\
\text { Woche } 24: 44 \%\end{array}$ \\
\hline $\begin{array}{l}\text { Langzeitwirksam- } \\
\text { keit (PASI 75) }\end{array}$ & Woche 50: $60 \%$ & & $\begin{array}{l}40 \mathrm{mg} \text { alle } 14 \text { Tage: } \\
\text { Woche } 60: \sim 56 \%\end{array}$ & Woche 52: 50\% \\
\hline
\end{tabular}

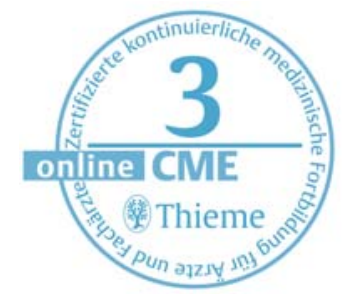

Psoriasis (und Psoriasisarthritis) wurden monoklonale Antikörper, Fusionsproteine und Zytokine in klinischen Studien erprobt. Bei den therapeutisch eingesetzten monoklonalen Antikörpern unterscheidet man chimäre Antikörper (Endung auf -ximab), humanisierte Antikörper (Endung auf -zumab) und vollständig humane Antikörper (Endung auf -umab). Bei chimären Antikörpern sind die variablen Regionen murinen Ursprungs, während bei humanisierten Antikörpern nur noch die Complementarity determining Regions der variablen Regionen murinen Ursprungs sind. Humane Antikörper beinhalten keine murinen Bestandteile mehr. Fusionsproteine (Endung auf -cept) bestehen häufig aus einem natürlich vorkommenden Bindungspartner des Zielmoleküls, zum Beispiel einem Zytokinrezeptor, der an die konstante Region eines Immunglobulin $\mathrm{G}$ (IgG) gekoppelt ist.

Aktuell besteht in den Mitgliedsländern der Europäischen Union (EU) eine Zulassung zur Second-Line-Therapie für erwachsene Patienten mit mittelschwerer bis schwerer Plaque-Psoriasis für die TNF $\alpha$-Antagonisten Adalimumab, Etanercept und Infliximab sowie für den AntiCD11a-Antikörper Efalizumab. Hierbei bedeutet Second-Line, dass eine Therapie mit CyA, MTX und oraler PUVA unwirksam war, kontraindiziert ist oder wegen Nebenwirkungen abgesetzt werden musste.

\section{TNF $\alpha$-Antagonisten}

TNF $\alpha$-Antagonisten beeinflussen chronisch-entzündliche Erkrankungen, die durch eine Überproduktion von TNF $\alpha$ charakterisiert sind, durch hemmende, neutralisierende und/oder zytotoxische Effekte. Die wichtigsten Eigenschaften der aktuell zur Therapie der Plaque-Psoriasis zugelassenen TNF-Antagonisten Adalimumab, Etanercept und Infliximab sind in Tab. 1 zusammengefasst. Bei grundsätzlich verwandtem Wirkprinzip, nämlich der Antagonisierung von TNF $\alpha$ (bei Etanercept auch TNF $\beta /$ Lymphotoxin), aus dem Gemeinsamkeiten bei Wirkungen und Nebenwirkungen abgeleitet werden können, bestehen auch Unterschiede zwischen Infliximab, Etanercept und Adalimumab. Bei Adalimumab handelt es sich um einen humanen Antikörper gegen TNF $\alpha$, der keine murinen Bestandteile enthält. Infliximab ist ein chimärer monoklonaler Antikörper (ca. 25\% murin). Bei Etanercept handelt es sich um ein Fusionsprotein aus je zwei p75-Molekülen des TNF-Rezeptors und dem konstanten Fragment von $\operatorname{IgG}_{1}$. Infliximab wird intravenös als Kurzinfusion über mindestens 2 Stunden verabreicht, Etanercept und Adalimumab werden subkutan injiziert.

Adalimumab und Etanercept werden subkutan, Infliximab intravenös verabreicht. 


\section{Wirksamkeit}

Alle 3 zugelassenen TNF-Antagonisten zeigen eine sehr gute Wirksamkeit in der Therapie der Psoriasis ( Tab. 1). Hierbei erzielen Infliximab und Adalimumab mit einem PASI75-Ansprechen bei bis zu $80 \%$ der Patienten bereits nach $10-12$ Wochen ein schnelleres und etwas besseres Ansprechen als Etanercept (PASI75 nach 12 Wochen je nach Dosierung zwischen 35\% und 50\%) (Beispiel für Therapieeffekt siehe $\bullet$ Abb. 2). In der Langzeittherapie nimmt die Wirksamkeit von Infliximab und Adalimumab etwas ab und gleicht sich innerhalb der Gruppe der TNF-Antagonisten an.

Bei Adalimumab und Infliximab zeigt sich ein rascherer Wirkungseintritt als bei Etanercept. Unter den TNF-Antagonisten zeigt Etanercept die kürzeste Halbwertszeit und kann so am besten gesteuert werden.

\section{Sicherheitsaspekte}

Sicherheitsaspekte schließen bei Infliximab das Auftreten von Infusionreaktionen vor allem vom Soforttyp ein, die wahrscheinlich nicht IgE-vermittelt und mit gegen Infliximab gerichteten Antikörpern (ATI) assoziiert sind ( Tab. 2). Hingegen sind Injektionsreaktionen vom Soforttyp bei Adalimumab und Etanercept selten. Bei Etanercept wird manchmal um die Einstichstelle ein juckendes Erythem, teilweise auch mit ödematöser Schwellung oder Papelbildung, beobachtet. Diese Injektionsreaktionen manifestieren sich bei den meisten Patienten innerhalb der ersten Behandlungswochen etwa 1-2 Tage nach der Injektion und halten ca. 3 - 5 Tage an. Mit der Gabe weiterer Injektionen nimmt die Intensität der Symptome in der Regel ab [7].

Von besonderer Bedeutung bei der Therapie mit TNF-Antagonisten ist das erhöhte Risiko für Infektionen. Hier spielen vor allem opportunistische Infektionen eine Rolle, insbesondere die Reaktivierung einer latenten Tuberkulose. Deswegen muss vor Beginn einer Therapie mit TNF-Blockern eine aktive oder latente Tuberkulose nach lokalen Richtlinien ausgeschlossen werden, wobei hier neben einer Anamnese bezüglich einer früheren Tuberkulose und eines Kontaktes mit an Tuberkulose erkrankten Personen auch die Röntgenuntersuchung des Thorax in 2 Ebenen sowie ein Mendel-Mantoux-Test ( Abb.3) gehören. Ferner sind Infektionen der Haut, vor allem Abszesse und Erysipele, sowie Infektionen der Atemwege bis hin zur Pneumonie relevant. In der Praxis sollte eine besondere Beobachtung und ggf. frühzeitige Therapie insbesondere von Patienten mit Verbrennungswunden oder anderen Hautdefekten erfolgen.

Anhand der aktuell verfügbaren Daten kann ein erhöhtes Risiko für maligne Tumore bei mit TNFAntagonisten behandelten Patienten weder be-

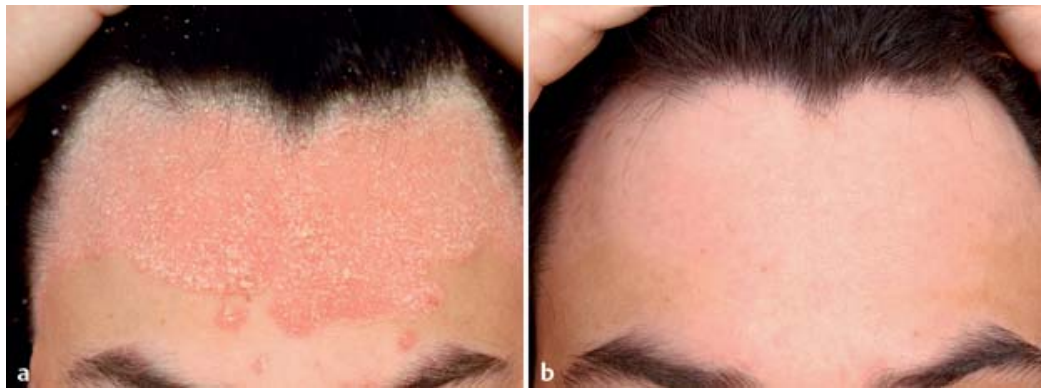

Abb. 2 Gesichtsbeteiligung bei einem Psoriasis-Patienten vor (a) und nach 8 Wochen (b) einer Etanercept-Therapie.

Tab. 2 Charakteristika von gegen Biologika gerichtete Antikörper ${ }^{\mathrm{a}}$.

\begin{tabular}{|c|c|c|c|c|}
\hline & Infliximab & Etanercept & Adalimumab & Efalizumab \\
\hline $\begin{array}{l}\text { Häufigkeit von } \\
\text { gegen Biologika } \\
\text { gerichtete } \\
\text { Antikörper }\end{array}$ & $\sim 2-43 \%$ & $\sim 0-6 \%$ & $\sim 0,6-17 \%$ & $\sim 6,3 \%$ \\
\hline $\begin{array}{l}\text { Neutralisierend } \\
\text { in vitro }\end{array}$ & Ja & Nein & Ja & $\begin{array}{l}\text { Nicht } \\
\text { untersucht }\end{array}$ \\
\hline $\begin{array}{l}\text { Auswirkung auf } \\
\text { Wirksamkeit }\end{array}$ & $\begin{array}{l}\text { Verminderung } \\
\text { der Wirksam- } \\
\text { keit }\end{array}$ & Nein & $\begin{array}{l}\text { Titerabhän- } \\
\text { gige Vermin- } \\
\text { derung der } \\
\text { Wirksamkeit }\end{array}$ & Nein \\
\hline $\begin{array}{l}\text { Auswirkung auf } \\
\text { Sicherheit }\end{array}$ & $\begin{array}{l}\text { Erhöhtes Risiko } \\
\text { für Infusions- } \\
\text { reaktionen }\end{array}$ & Nein & Nein & Nein \\
\hline
\end{tabular}

a Die Daten entstammen teilweise dem Einsatz der Biologika in anderen Indikationen als der Plaque-Psoriasis.

stätigt noch ausgeschlossen werden [8]; es erfolgen diesbezüglich weiterhin regelmäßige Auswertungen von Registerdaten.

Ferner kann es in seltenen Fällen unterTNF-Antagonisten zu mit Anti-dsDNS-Antikörpern einhergehenden Lupus-erythematodes-artigen Hautveränderungen kommen, die in etwa der Hälfte der Fälle auf die Haut beschränkt sind [9]. Häufiger kommt es zum Auftreten meist niedrig titriger antinukleärer Antikörper, ohne dass klinische Symptome im Sinne eines Lupus erythematodes vorliegen. In diesen Fällen kann die Therapie mit TNF-Antagonisten ohne Veränderungen fortgeführt werden.

Während der Therapie mit TNF-Antagonisten kann es ferner zur Verschlechterung einer Herzinsuffizienz und zu Leberwerterhöhungen, letzteres vor allem unter Infliximab, kommen.

In Einzelfällen wurde bei Patienten, die wegen anderer Indikationen mit TNF-Antagonisten behandelt wurden, die Erstmanifestation einer Psoriasis vulgaris oder pustulösen Psoriasis beobachtet. Auch bei der Therapie der Psoriasis vulgaris ist das Neuauftreten einer pustulösen Variante der Psoriasis in einzelnen Fällen beschrieben worden [10]. Pharmakologische Arzneimittelinteraktionen sind bei TNF-Antagonisten nicht relevant.

Opportunistische Infektionen treten unter TNFAntagonisten vermehrt auf.

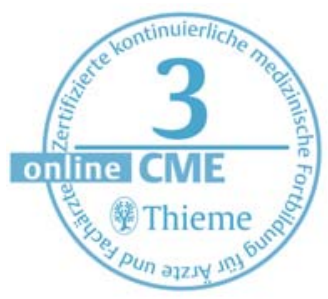


Abb. 3 Durchführung des Mendel-Mantoux-Hauttests vor Beginn einer Therapie mit TNFBlockern als Teil der Maßnahmen zum Ausschluss einer latenten oder aktiven Tuberkulose. Findet sich beim Mendel-Mantoux-Test eine Induration, die einen Durchmesser $<5 \mathrm{~mm}$ besitzt, sollte der Test am selben Tag am kontralateralen Arm wiederholt werden. Eine Induration von $\geq 5 \mathrm{~mm}$ wird als positiv gewertet. Zur Durchführung des MendelMantoux-Tests steht aktuell das Präparat Tuberkulin PPD RT23 SSI, 2 Tuberkulineinheiten (T.E.) $/ 0,1 \mathrm{ml}$, (Statens-SerumInstitut, Kopenhagen) zur Verfügung. Hiervon werden $0,1 \mathrm{ml}$ $\approx 2 \mathrm{~T}$. E. intrakutan gespritzt.

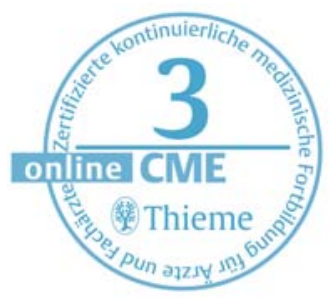

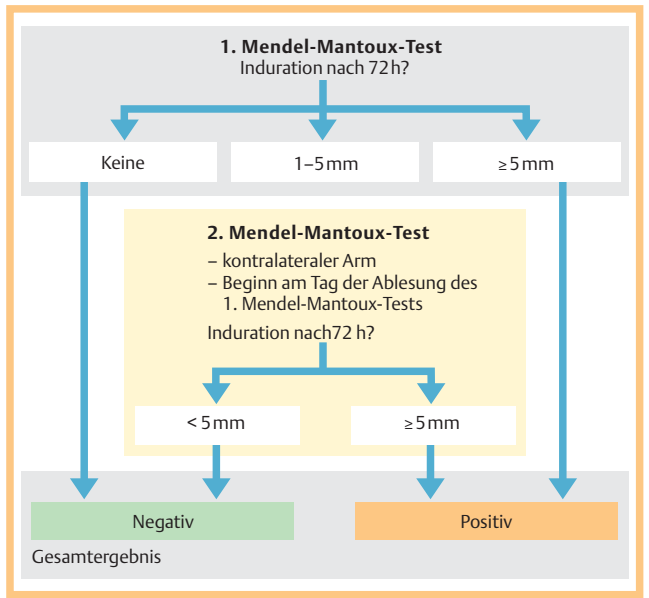

\section{Efalizumab}

Efalizumab ist ein humanisierter Antikörper, der gegen CD11a gerichtet ist, das die $\alpha$-Untereinheit des Adhäsionsmoleküls LFA-1 (Lymphocyte function-associated Antigen 1) darstellt. Efalizumab kann die Interaktion von LFA-1 mit ICAM-1 (Intercellular Adhesion Molecule 1) hemmen und reduziert dadurch die Migration von T-Zellen in die Haut und wahrscheinlich auch die T-ZellAktivierung.

Efalizumab ist seit 2003 in den USA und seit 2004 in den Mitgliedsländern der Europäischen Union (EU) zugelassen, wobei die chronische PlaquePsoriasis bei Erwachsenen bisher die einzige zugelassene Indikation darstellt. Nach Schätzungen der Hersteller wurden bisher mehr als 50000 Patienten weltweit mit Efalizumab behandelt, mit einer Therapiedauer von mehr als 40000 Patientenjahren.

Nach Drucklegung dieses Artikels empfahl die EMEA ein Aussetzen der Zulassung von Efalizumab (Raptiva $\left.{ }^{\circledR}\right)$, nachdem das Nutzen-Risiko-Verhältnis aufgrund neuer Meldungen über überwiegend neurologische unerwünschte Ereignisse als ungünstig bewertet wurde.

\section{Wirksamkeit}

Efalizumab wird einmal wöchentlich subkutan verabreicht, bei Erstgabe in einer Dosis von $0,7 \mathrm{mg} / \mathrm{kg}$, danach in einer Dosis von $1 \mathrm{mg} / \mathrm{kg}$. In kontrollierten klinischen Studien mit Efalizumab erzielten zwischen $22 \%$ und 39\% der Patienten nach 12 Wochen eine PASI75-Antwort verglichen mit $2 \%-5 \%$ in der Placebogruppe. Bei Weiterführung der Therapie stieg die Ansprechrate im Verlauf noch weiter bis auf $50 \%$ an [11].

\section{Sicherheitsaspekte}

Der häufigste unerwünschte Effekt einer Therapie mit Efalizumab (ca. 3\% murin) ist eine grippeähnliche Reaktion nach den ersten beiden Injektionen: nach der ersten Injektion bei ca. 30\% der mit Efalizumab (1 mg/kg) behandelten Patienten (Placebo: ca. 15\%), die aber unter der reduzierten Initialdosis von $0,7 \mathrm{mg} / \mathrm{kg}$ seltener und meist nur leicht bis mäßig ausgeprägt ist [12]. Andere Symptome einer Überempfindlichkeitsreaktion wurden in klinischen Studien bei mit Efalizumab behandelten Patienten (ca. 8\%) kaum häufiger als bei mit Placebo behandelten Patienten beobachtet (ca. 7\%) [12].

Ferner kann es zu Veränderungen des Blutbilds kommen. Eine Lymphozytose, die häufig während des Therapiezeitraums bestehen bleibt, wird bei bis $\mathrm{zu} \mathrm{40 \%} \mathrm{der} \mathrm{Patienten} \mathrm{beobachtet}$ und ist wahrscheinlich die Folge der Hemmung der Lymphozyten-Extravasation. Eine Thrombozytopenie $(<50000 / \mu l)$ trat bei etwa $0,3 \%$ der Patienten in klinischen Studien auf, die Efalizumabtherapie muss dann abgesetzt werden [13]. In klinischen Studien traten auch Infektionen unter Efalizumab etwas häufiger als in der Placebogruppe auf. Ferner wurden unter der Therapie mit Efalizumab Einzelfälle von seltenen neurologischen Erkrankungen wie des Guillain-BarréSyndrom (akute Polyradikuloneuropathie) oder der transversalen Myelitis beobachtet. Kürzlich wurde von drei gesicherten und einem Verdachtsfall einer progressiven multifokalen Leukenzephalopathie (PML) bei Psoriasispatienten unter Efalizumab berichtet, wobei die drei Patienten mit der gesicherten Diagnose der PML über mehr als drei Jahre mit Efalizumab behandelt wurden. Bei der PML handelt es sich um eine häufig tödlich verlaufende entzündliche demyelinisierende Erkrankung des zentralen Nervensystems, deren erste Symptome motorische, sensorische oder kognitive Störungen wie Paresen, Gefühlsstörungen oder eine Aphasie sein können. Die PML tritt fast ausschließlich bei immunsupprimierten Menschen auf und wird hervorgerufen durch eine Reaktivierung des JohnCunningham (JC)-Virus, einem Polyomavirus, der eine hohe Durchseuchung (> 80\%) in der Bevölkerung aufweist. Eine erhöhte Inzidenz maligner Erkrankungen wurde in einer aktuellen Analyse klinischer Studien nicht beobachtet [14], jedoch liegen für eine sichere Beurteilung noch keine ausreichenden Langzeitbeobachtungen vor.

Unter Efalizumab kann es zu Blutbildveränderungen kommen.

\section{Psoriasiforme Hautveränderungen und Verschlechterung der Psoriasis}

Bei den während einer Therapie mit Efalizumab möglicherweise auftretenden psoriasiformen Hautveränderungen werden 2 klinische Bilder unterschieden, die als Localized mild Breakthrough und Generalized inflammatory Flare bezeichnet werden [15]. 
Localized mild Breakthrough. Beim Localized mild Breakthrough handelt es sich um entzündliche Papeln und Plaques, die häufig an vorher nicht betroffenen Körperarealen auftreten, bevorzugt an Hals, Rumpf und an den Körperbeugen. Histologisch zeigt sich ein psoriasiformes Bild mit Infiltraten von Neutrophilen. Da die vorbestehenden Hautveränderungen der Psoriasis oftmals eine Besserung zeigen, trifft die Bezeichnung „Verschlechterung der Psoriasis“ für dieses Phänomen meist nicht zu und der Zusammenhang mit der Grunderkrankung bedarf weiterer Klärung. Ein Localized mild Breakthrough tritt bei bis zu 30\% der behandelten Patienten auf, typischerweise zwischen der 4 . und 8. Behandlungswoche. In vielen Fällen erfolgt eine Besserung spontan oder unter einer zusätzlichen Lokaltherapie mit Kortikosteroiden. Eine Therapieunterbrechung ist in der Regel nicht erforderlich.

Generalized inflammatory Flare. Hingegen tritt der Generalized inflammatory Flare seltener auf, schätzungsweise bei $1-3 \%$ der behandelten Patienten. Es handelt sich um eine Verschlechterung der Psoriasis mit Auftreten stark entzündlicher Läsionen innerhalb vorbestehender Plaques, wobei zusätzlich auch zuvor unbefallene Körperareale betroffen sein können. Eine spontane Besserungstendenz besteht typischerweise nicht. Bei Patienten, die zuvor auf die Therapie mit Efalizumab angesprochen haben, kann versucht werden, die Exazerbation zwischenzeitlich durch eine zusätzliche Systemtherapie abzufangen und diese anschließend wieder abzusetzen. Ansonsten ist eine Umstellung auf eine andere Systemtherapie, z.B. auf einen TNF-Antagonisten, sinnvoll.

Rebound. Nach Beendigung der Therapie mit Efalizumab kann es zu einem Rebound kommen, d.h. zu einer Verschlechterung der Psoriasis über den Ausgangszustand hinaus. Meist tritt eine Verschlechterung der Plaque-Psoriasis auf, aber es können ebenfalls eine Erythrodermie oder eine pustulöse Psoriasis auftreten [15]. In diesen Fällen ist eine systemische Therapie angezeigt.

Psoriasisarthritis. Es ist bisher nicht sicher, wie sich eine Therapie mit Efalizumab auf das Auftreten und den Verlauf einer Psoriasisarthritis auswirkt. Einerseits wurde in einer 12-wöchigen, randomisierten Studie bei Patienten mit Psoriasisarthritis $(n=107)$ das primäre Zielkriterium in der Efalizumabgruppe mit 28\% häufiger als in der Placebogruppe mit 19\% erreicht, der Unterschied war jedoch nicht statistisch signifikant [16]. Andererseits gibt es eine Reihe von Einzellfallbeobachtungen und Fallserien, die das Neuauftreten einer zum Teil schweren Psoriasisarthritis unter Efalizumab beschrieben, wobei die Hautläsionen bei einigen dieser Patienten unter Efalizumab gut kontrolliert waren $[17,18]$. Auf jeden Fall ist Efali- zumab zur Therapie der Psoriasis-Arthritis nicht zugelassen.

Ein Localized mild Breakthrough tritt bei bis zu 30\% der Patienten vorübergehend auf und führt in der Regel nicht zu einem Therapieabbruch.

\section{Antikörperbildung}

Da es sich bei den TNF-Antagonisten und bei Efalizumab nicht um körpereigene Proteine handelt, kann es grundsätzlich zu einer Bildung von Antikörpern gegen das Fremdprotein kommen. Wie häufig solche Antikörper auftreten, ist bisher nicht geklärt. Zum einen hängt die gefundene Häufigkeit von Begleittherapien (niedriger bei gleichzeitiger Therapie mit MTX), der Therapiedauer und wahrscheinlich auch von der behandelten Erkrankung ab. Aufgrund unterschiedlicher Messverfahren und methodischer Schwierigkeiten können verschiedene Studien aktuell nicht verglichen werden. Entscheidend für die Bedeutung der Antikörper ist vor allem, ob die Antikörperbildung mit einer verminderten Wirksamkeit einhergeht, was für Adalimumab und Infliximab der Fall ist, und ob es gehäuft zu Nebenwirkungen kommt, was bisher nur für Infusionsreaktionen bei Infliximab beobachtet wurde (O Tab. 2).

\section{Systemische Therapie in Schwanger- schaft und Stillzeit \\ $\nabla$}

Kein Systemtherapeutikum ist zur Therapie der Psoriasis in der Schwangerschaft oder Stillzeit zugelassen. Bei Retinoiden und MTX ist eine teratogene Wirkung beim Menschen gesichert. Das empfohlene Mindestintervall zwischen Therapieende und Schwangerschaft beträgt für Acitretin 2 Jahre. Während dieses Zeitraumes wird eine sichere (doppelte) Kontrazeption empfohlen. Eine Minipille sollte unter Acitretin wegen der Gefahr einer verminderten Wirksamkeit nicht verwendet werden. Schwangerschaftstests sind vor einer Therapie mit Acitretin und anschließend monatlich erforderlich.

Cave: Unter Acitretin keine Minipille, Schwangerschaftstest vor Therapiebeginn und anschließend monatlich.

Bei MTX sollte der Zeitraum zwischen Therapieende und Schwangerschaft mindestens 3 bis 6 Monate betragen. Hingegen wurde für CyA bisher weder im Tierversuch noch beim Menschen eine teratogene Wirkung nachgewiesen, jedoch scheint CyA das Risiko schwangerschaftsspezifischer Komplikationen wie einer Präeklampsie

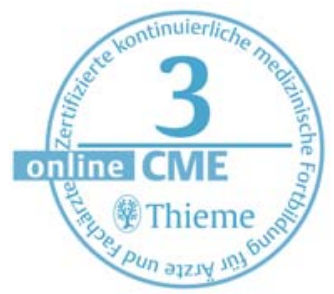


Abb. 4 Jahrestherapiekosten der Erhaltungstherapie. Es wurde von einem $80 \mathrm{~kg}$ schweren Patienten ausgegangen. Für die Biologika wurden die Kosten für die zugelassenen Dosen berechnet. Bei CyA wurden die Kosten ausgehend von einer Dosis von $4 \mathrm{mg} / \mathrm{kg}$ $\mathrm{KG} /$ Tag, bei Fumaderm ${ }^{\circledR}$ von 4 Tabletten/Tag, bei Acitretin von $0,5 \mathrm{mg} / \mathrm{kg} \mathrm{KG} /$ Tag und bei Methotrexat von $20 \mathrm{mg} /$ Woche als orale Therapie bestimmt.

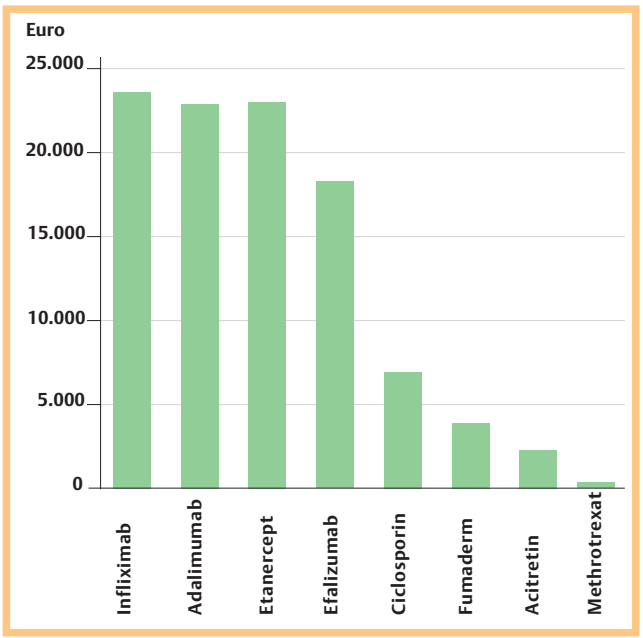

zu erhöhen. Bei Fumarsäureestern fand sich kein teratogenes Potenzial, aber hier gibt es keine ausreichenden Erfahrungen zur Therapie bei schwangeren Frauen. Weder bei CyA noch bei Fumarsäureestern wird ein therapiefreies Intervall zwischen Therapieende und Beginn der Schwangerschaft gefordert.

Bei TNF-Blockern ist bisher in Tierversuchen keine Teratogenität nachgewiesen worden, und Schwangerschaften unter TNF-Blockern wiesen keinen unerwarteten Ausgang auf. Aufgrund der unzureichenden Datenlage und der immunsuppressiven Wirkung auf die Mutter und möglicherweise auch auf das Kind sind TNF-Blocker während der Schwangerschaft nicht zugelassen. Das empfohlene Mindestintervall zwischen Therapieende und Schwangerschaft beträgt laut Fachinformation für Infliximab 6 Monate, für Adalimumab 5 Monate, für Etanercept finden sich hierzu in der Fachinformation keine Angaben. Patientinnen, die eine Efalizumabtherapie erhalten, sollten ebenfalls eine adäquate Verhütungsmethode anwenden; ein Intervall zwischen Therapieende und Konzeption wird in der Fachinformation ebenfalls nicht angegeben.

Sollten eine Lokaltherapie und eine UV-Therapie während der Schwangerschaft keine ausreichende Wirkung zeigen und eine systemische Therapie aufgrund der Schwere des Hautbefalls unbedingt erforderlich sein, ist wahrscheinlich CyA aufgrund der vielfältigen Erfahrungen in der Transplantationsmedizin der Vorzug zu geben.

Keines der Systemtherapeutika ist während der Schwangerschaft oder in der Stillzeit zugelassen.

Sowohl Acitretin, MTX als auch CyA gehen in die Muttermilch über. Bei CyA ist ferner zu beachten, dass zusätzlich auch der in der Kapsel enthaltene Alkohol in die Muttermilch übergeht. Es ist nicht bekannt, ob Fumarsäureester in die Muttermilch übergehen. Sie sind während der Stillzeit nicht zugelassen. Es wurde bei einer Patientin gezeigt, dass Etanercept in die Muttermilch übergeht, bei Adalimumab, Infliximab und Efalizumab liegen hierzu keine Daten vor. Allerdings ist eine Sekretion in die Muttermilch zu erwarten, da Immunglobuline in die Muttermilch übergehen, und diese Biologika sollen daher während der Stillzeit ebenfalls nicht angewendet werden.

\section{Systemtherapien bei Männern mit Kinderwunsch}

Männer, die MTX einnehmen, sollten während und bis 3 Monate nach der Einnahme eine sichere Kontrazeption durchführen. Wichtig ist auch, Männer darauf hinzuweisen, dass es auch zu persistierender Oligospermie kommen kann [19]. Eine Therapie mit Acitretin, CyA oder Fumarsäureestern kann auch bei Kinderwunsch des Mannes weitergeführt werden.

Zur Wirkung von TNF-Antagonisten auf die Spermatogenese gibt es kaum Daten. Bisher wurden keine Veränderungen der Fertilität beschrieben. Eine kürzlich durchgeführte Untersuchung zeigte einen Trend zu verringerter Motilität der Spermien sowie eine signifikante Abnahme der ovalen Spermien bei Patienten mit Morbus Crohn unter Infliximab [20], wobei ein solcher Befund nicht unbedingt mit einer Beeinträchtigung der Zeugungsfähigkeit einhergehen muss. Auch aufgrund fehlender Hinweise auf mutagene Eigenschaften von TNF-Antagonisten wird weiterhin diskutiert, ob es für Männer mit Kinderwunsch notwendig ist, eine Therapie mit TNF-Anatagonisten zu unterbrechen. Zur Therapie mit Infliximab, Adalimumab und Etanercept sind für Männer in der Fachinformation keine Einschränkungen bezüglich der Zeugung von Kindern enthalten. Auch bei Efalizumab bestehen bei ebenfalls unzureichender Datenlage diesbezüglich in der Fachinformation keine Vorgaben.

Acitretin, Fumarsäureester und CyA dürfen von Männern mit Kinderwunsch eingenommen werden.

\section{Therapiekosten}

$\nabla$

Die Therapiekosten der medikamentösen Systemtherapien der Psoriasis unterscheiden sich stark zwischen den Medikamenten (s. $\bullet$ Abb. 4). MTX ist das günstigste Medikament mit jährlichen Jahrestherapiekosten meist unter 200 Euro, gefolgt von Acitretin, Fumaderm ${ }^{\circledR}$ und CyA. Efalizumab ist unter den Biologika das günstigste Medikament mit Jahrestherapiekosten von etwa 18000 Euro, während die Kosten für die TNF-Antagonisten in der Erhaltungstherapie bei etwa 23000 Euro liegen. In der Induktionsphase kommen bei Infliximab aufgrund der häufigeren Applikation (Woche 0, 2 und 6) noch weitere Kosten 
hinzu, ebenso bei Etanercept, sofern es in den ersten 12 Wochen in einer Dosis von $2 \times 50 \mathrm{mg} /$ Woche verabreicht wird.

\section{Zusammenfassung}

Die Psoriasis vulgaris ist eine chronisch entzündliche Dermatose mit einer Prävalenz von $2-3 \%$ in der Bevölkerung. Die Psoriasis-Arthritis, die bei etwa $10-20 \%$ der Psoriasis-Patienten auftritt, ist als extrakutane Manifestationsform seit längerem bekannt. In den vergangenen Jahren wurden außerdem die zum Teil starke psychosoziale Belastung der Psoriasis-Patienten sowie erhebliche krankheitsbedingte Einschränkungen der Lebensqualität dokumentiert. Darüber hinaus liegen zunehmend Erkenntnisse darüber vor, dass Psoriasis-Patienten gehäuft an anderen, vorwiegend metabolischen und kardiovaskulären Erkrankungen leiden. Parallel zu dem zunehmenden Wissen über Komorbiditäten der Psoriasis haben sich die Möglichkeiten für Systemtherapien in den letzten Jahren durch die Zulassung von Biologika erweitert.

\section{Interessenkonflikt}

$\nabla$

PD Dr. med. R. Mössner hat Reisestipendien von Abbott, Essex und Wyeth erhalten und war bezahlte Referentin für Abbott, Wyeth und Serono. Prof. Dr. med. M. P. Schön hat Vertragshonorare von Abbott, Essex, Merck Serono und Wyeth erhalten.

\section{Literatur}

1 Nast A, Kopp IB, Augustin M, Banditt KB et al. S3-Guidelines for the therapy of psoriasis vulgaris. J Dtsch Dermatol Ges 2006; 4 Suppl 2: S1 - S126

2 Augustin M, Kruger K, Radtke MA et al. Disease severity, quality of life and health care in plaque-type psoriasis: a multicenter cross-sectional study in Germany. Dermatology 2008; 216: $366-372$

3 Altmeyer P, Hartwig R, Matthes U. Efficacy and safety profile of fumaric acid esters in oral long-term therapy with severe treatment refractory psoriasis vulgaris. A study of 83 patients. Hautarzt 1996; 47: $190-196$
4 Goldfarb MT, Ellis CN, Gupta AK et al. Acitretin improves psoriasis in a dose-dependent fashion. J Am Acad Dermatol 1988; 18: 655-662

5 Flytstrom I, Stenberg B, Svensson A et al. Methotrexate vs. ciclosporin in psoriasis: effectiveness, quality of life and safety. A randomized controlled trial. $\mathrm{Br}$ J Dermatol 2008; 158: $116-121$

6 Saurat JH, Stingl G, Dubertret L et al. Efficacy and safety results from the randomized controlled comparative study of adalimumab vs. methotrexate vs. placebo in patients with psoriasis (CHAMPION). $\mathrm{Br} J$ Dermatol 2008; 158: $558-566$

7 Zeltser R, Valle L, Tanck C et al. Clinical, histological, and immunophenotypic characteristics of injection site reactions associated with etanercept: a recombinant tumor necrosis factor alpha receptor: Fc fusion protein. Arch Dermatol 2001; 137: 893 - 899

8 Askling J, Bongartz T. Malignancy and biologic therapy in rheumatoid arthritis. Curr Opin Rheumatol 2008; 20: $334-339$

9 De Bandt M, Sibilia J, Le L et al. Systemic lupus erythematosus induced by anti-tumour necrosis factor alpha therapy: a French national survey. Arthritis Res Ther 2005; 7: R545-R551

10 Mossner R, Thaci D, Mohr J et al. Manifestation of palmoplantar pustulosis during or after infliximab therapy for plaque-type psoriasis: report on five cases. Arch Dermatol Res 2008; 300: 101 - 105

11 Leonardi C, Menter A, Hamilton Tet al. Efalizumab: results of a 3-year continuous dosing study for the long-term control of psoriasis. Br J Dermatol 2008; 158: 1107 1116

12 Scheinfeld N. Efalizumab: a review of events reported during clinical trials and side effects. Expert Opin Drug Saf 2006; 5: 197 - 209

13 Fachinformation Raptiva 100 mg/ml; Stand 07/08. Serono Europe Ltd, London, Vereinigtes Königreich

14 Leonardi CL, Toth D, Cather JC et al. A review of malignancies observed during efalizumab (Raptiva) clinical trials for plaque psoriasis. Dermatology 2006; 213: 204-214

15 Carey W, Glazer S, Gottlieb AB et al. Relapse, rebound, and psoriasis adverse events: an advisory group report. J Am Acad Dermatol 2006; 54: S171 - S181

16 Papp KA, Caro I, Leung HM et al. Efalizumab for the treatment of psoriatic arthritis. J Cutan Med Surg 2007; 11: 57- 66

17 Viguier M, Richette P, Aubin F et al. Onset of psoriatic arthritis in patients treated with efalizumab for moderate to severe psoriasis. Arthritis Rheum 2008; 58: $1796-$ 1802

18 Myers WA, Najarian D, Gottlieb AB. New-onset, debilitating arthritis in psoriasis patients receiving efalizumab. J Dermatolog Treat 2006; 17: 353 - 354

19 Sussman A, Leonard JM. Psoriasis, methotrexate, and oligospermia. Arch Dermatol 1980; 116: 215-217

20 Mahadevan U, Terdiman JP, Aron J et al. Infliximab and semen quality in men with inflammatory bowel disease. Inflamm Bowel Dis 2005; 11: 395-399

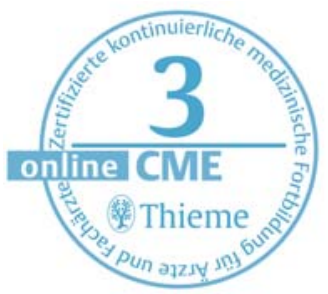




\section{CME-Fragen Medikamentöse System- therapien der Psoriasis}
1
A Small Molecule.
B Fusionsprotein.
C chimärer Antikörper.
D humaner Antikörper.
E Antihypertensivum.

2 Eine Teratogenität ist für folgende Medikamente nachgewiesen.

A Efalizumab

B Acitretin und Fumaderm

C Methotrexat und Fumaderm

D Acitretin und Methotrexat

E Fumaderm und Etanercept

\section{3}

$\begin{array}{ll}\text { A } & \text { Acitretin } \\ \text { B } & \text { Fumaderm } \\ \text { C } & \text { Methotrexat } \\ \text { D } & \text { Cyclosporin A } \\ \text { E } & \text { Etanercept }\end{array}$

4 Welche Antwort ist richtig?

1 Es treten keine Antikörper gegen Adalimumab auf, weil

2 Adalimumab ein humaner Antikörper ist.

A Aussage 1 ist richtig, Aussage 2 ist falsch.

B Aussage 1 ist falsch, Aussage 2 ist richtig.

C Aussage 1 und 2 sind richtig und die Verknüpfung ist richtig.

D Aussage 1 und 2 sind richtig, aber die Verknüpfung ist falsch.

E Aussagen 1 und 2 sind falsch.

\section{Der Localized mild Breakthrough}

A stellt eine Nebenwirkung von Etanercept dar.

B zwingt in der Regel zum Therapieabbruch.

C tritt vor allem bei Vorhandensein von Antikörpern gegen das systemische Antipsoriatikum auf.

D tritt vor allem in zuvor nicht von Psoriasisherden betroffenen Hautarealen auf.

E tritt vor allem nach Absetzen von Efalizumab auf.

6

A bei $75 \%$ der Patienten die Psoriasis abgeheilt ist.

B der PASI sich um $75 \%$ verglichen mit dem Ausgangsbefund gebessert hat.

C der PASI sich um mindestens $75 \%$ verglichen mit dem Ausgangsbefund gebessert hat.

D der PASI nur noch $75 \%$ des Ausgangsbefundes beträgt.

E der PASI maximal $75 \%$ des Ausgangsbefundes beträgt.

7

$\begin{array}{ll}\text { A } & \text { Fumaderm } \\ \text { B } & \text { Cyclosporin A } \\ \text { C } & \text { Adalimumab } \\ \text { D } & \text { Etanercept } \\ \text { E } & \text { Efalizumab }\end{array}$

8 Bei welchem der Medikamente dürfen Frauen unmittelbar nach Absetzen der Therapie schwanger werden? Fumaderm

Acitretin

Methotrexat

Adalimumab

Infliximab

Welche Nebenwirkung ist nicht typisch für Fumaderm ${ }^{\circledR}$ ?

A Flush

B Lymphopenie

C Eosinophilie

D Leberzirrhose

E Magenschmerzen

\section{0}

A

B Acitretin

C Cyclosporin A

D Methotrexat

E Efalizumab

Dieser Artikel wurde geändert gemäß folgendem Erratum vom 14. 1. 2010:

Im Kapitel „Systemtherapien bei Männern mit Kinderwunsch“ muss es heißen „Zur Therapie mit Infliximab, Adalimumab und Etanercept sind für Männer in der Fachinformation keine Einschränkungen bezüglich der Zeugung von Kindern enthalten“.
Die CME-Frage 10 sowie die Antwortmöglichkeiten wurden entsprechend geändert.

Der Verlag entschuldigt sich für das Versehen. 